Pacific Journal of Mathematics

BERGMAN KERNEL FUNCTIONS AND THE THREE TYPES OF 


\title{
BERGMAN KERNEL FUNCTIONS AND THE THREE TYPES OF CANONICAL DOMAINS
}

\author{
Shozo Matsuura
}

\begin{abstract}
The objects of this paper are to extend J. Mitchell's theorems on minimal domains of moment of inertia for sufficiently wider class $\mathscr{F}$ and to discuss the relations among the three types of canonical domains in the $\mathscr{F}$-equivalent class. Some of the results are that, (i) a domain $D$ is the minimal domain of moment of inertia of the $\left[0, I_{n} ; 0\right]^{D_{-}}$. equivalent class if and only if the following holds:

$$
M_{D}^{0 I}(Z, 0)=Z \text { for } Z \in D,
$$

where $M_{D}^{0 I}{ }^{n}(Z, 0)$ is the minimizing function of the $\left(0, I_{n} ; 0\right)_{D^{-}}$class, and (ii) if $A, B$ and $C$ are the sets of Bergman's minimal domains, Bergman's representative domains and Mitchell's minimal domains of moment of inertia with the same center in the -equivalent class respectively, and if any one of the three relations $A \cap B \neq \phi, A \cap C \neq \phi$ and $B \cap C \neq \phi$ holds, then it follows that $A \supset B=C$.
\end{abstract}

J. Mitchell [5] has recently proved the theorems of minimal moment of inertia for complete circular domains in the space $C^{n}$. In our paper [10] we have extended them for bounded Bergman's minimal domains by making use of the Bergman kernel function. But there are two restrictions which can be removed. One of them is that the transformations $W=F(Z)$ considered must belong to the class $\mathscr{F}_{0}$ (the set of holomorphic mappings which preserve the volume of the initial domain), and another is that the domains considered must be Bergman's minimal domains.

In $\S 4$ of this paper we shall remove these two restrictions and extend the theorems of minimal moment of inertia for wider class $\mathscr{F}$ of transformations without volume preserving property.

In the case of several complex variables, the analogue of Riemann's mapping theorem does not hold even for simply connected domains. As the canonical domain corresponding to the unit circle in Riemann's mapping theorem, the representative domain is introduced by $\mathrm{S}$. Bergman. Another object of this paper is to discuss the relations among the three types of canonical domains, i.e., Bergman's minimal domains, Bergman's representative domains and the minimal domains of moment of inertia (abbreviated as the moment minimal domains) in wider class $\mathscr{F}[\S 3, \S 4]$.

The main results are Theorem 5 in $\S 3$ and Theorems 10 and 12 in $\S 4$. 
Our theorems obtained in this paper can be extended to the cases of $m$-minimal domains, $m$-representative domains [3], [4], [10] and $m$-moment minimal domains [10] $(m \geqq 1)$. Therefore, for simplicity, we treat the case of $m=1$, which is our case.

In $\S$ 's 1,2 and 3 we treat minimal, representative and moment minimal domains and the relations among them under the restricted initial conditions of transformations. In $\S 4$ we shall discuss the three types of canonical domains mentioned above under the extended class $\mathscr{F}$ and in particular, we attach importance to the moment minimal problem.

1. Preliminaries. In various extremal problems, mapping functions which may be meromorphic or many-valued can be successfully used. In order to treat such problems, we must extend the concept of a domain and its Bergman kernel function.

We assume that each domain with which we deal is a generalized domain (which is called a "domain" hereafter) in $C^{n}$ following M. Maschler [3, p. 503], [4, pp. 765-770], which can be mapped holomorphically onto a bounded univalent domain in $C^{n}$. By a holomorphic mapping (or holomorphic vector function) of a domain $D$ onto a domain $\Delta$ we mean a one-to-one mapping which, except in a denumerable number of analytic segments of manifolds of complex dimensions $\leqq n-1$, can be described locally in the column vector

$$
W=W(Z) \equiv\left(w_{1}(Z), w_{2}(Z), \cdots, w_{n}(Z)\right)^{\prime},
$$

where $w_{i}(Z)(i=1,2, \cdots, n)$ are holomorphic scalar functions of $Z \equiv\left(z_{1}, z_{2}, \cdots, z_{n}\right)^{\prime} \in D$ with a nonvanishing Jacobian. But, in accordance with the remarks of M. Maschler [3], [4], we allow $w_{i}(Z)$ $(i=1,2, \cdots, n)$ to be multi-valued meromorphic functions provided that the Jacobian $\operatorname{det}(d W(Z) / d Z)$ is a single-valued meromorphic function and does not vanish identically in $D$. In such a case we identify in the image domain $\Delta$ the points which correspond to the same point of $D$.

Hereafter, we shall use, except for Greek letters, upper-case letters for vectors and matrices and lower-case letters for scalars.

It is known that such domains $D$ and $\Delta$ possess Bergman kernel functions $k_{D}(Z, \bar{X}), Z, X \in D$ and $k_{d}(W, \bar{Y}), W, Y=W(X) \in \Delta$, and the relation

$$
k_{D}(Z, \bar{X})=k_{\Delta}(W, \bar{Y}) \operatorname{det} \frac{d W(Z)}{d Z} \overline{\operatorname{det} \frac{d W(X)}{d X}}
$$

holds. $k_{D}(Z, \bar{X})$ is holomorphic with respect to $Z$ and $\bar{X}$, and belongs to $\mathscr{L}^{2}(D)$ which is a class of single-valued holomorphic 
scalar functions $f(Z)$ square integrable in the sense of Lebesgue in $D$, namely

$$
(f, f)_{D} \equiv \int_{D}|f(Z)|^{2} d v_{Z}<+\infty,
$$

where $d v_{z}$ is the Euclidean volume element of the $Z$-space. If $D$ is a bounded domain, then $k_{D}(Z, \bar{Z})>0, Z \in D$, holds.

We first define the differentiation of matrix functions with respect to vector variables. Let $F(Z)$ be a matrix function

$$
F(Z) \equiv\left(\begin{array}{ccc}
f_{11}(Z) & \cdots & f_{1 m}(Z) \\
\vdots & & \vdots \\
f_{l 1}(Z) & \cdots & f_{l m}(Z)
\end{array}\right),
$$

where $f_{i j}(Z)(i=1,2, \cdots, l ; j=1,2, \cdots, m)$ are scalar differentiable functions of $Z \equiv\left(z_{1}, z_{2}, \cdots, z_{n}\right)^{\prime}$, and $d / d Z$ is the differential operator of row vector type:

$$
\frac{d}{d Z} \equiv\left(\frac{\partial}{\partial z_{1}}, \frac{\partial}{\partial z_{2}}, \cdots, \frac{\partial}{\partial z_{n}}\right)
$$

We define the differentiation as follows:

$$
\frac{d}{d Z} F(Z) \equiv \frac{d}{d Z} \times F(Z)
$$

Here the Kronecker product of two matrices $A$ and $B=\left(b_{i j}\right)$ (notation: $A \times B$ ) denotes

$$
A \times B=\left(\begin{array}{ccc}
A b_{11} & A b_{12} & \cdots \\
A b_{21} & A b_{22} & \cdots \\
\vdots & \vdots
\end{array}\right),
$$

where $A$ may be an operator of matrix type.

For convenience, we note here some differential formulas. Let the functions $A, B$ and $b$ of $Z \equiv\left(z_{1}, z_{2}, \cdots, z_{n}\right)^{\prime}$ be $(k \times l),(l \times m)$ matrices and a scalar, respectively. The following formulas can be easily calculated:

$$
\begin{aligned}
& \frac{d}{d Z} A B=\frac{d A}{d Z}\left(I_{n} \times B\right)+A \frac{d B}{d Z}, \\
& \frac{d A}{d Z}=\frac{d A}{d W}\left(\frac{d W}{d Z} \times I_{l}\right), \quad A=A(W), \\
& W \equiv\left(w_{1}(Z), w_{2}(Z), \cdots, w_{n}(Z)\right)^{\prime},
\end{aligned}
$$




$$
\frac{d A b}{d Z} \equiv \frac{d A \times b}{d Z}=\frac{d A}{d Z} \times b+A \times \frac{d b}{d Z},
$$

where $I_{k}$ denotes an identity matrix of order $k$ ( $k$ : positive integer).

Next we define the transposed conjugate differential operator $d / d Z^{*}$ as follows:

$$
\frac{d}{d Z^{*}} A(\bar{Z}) \equiv \frac{d}{d Z^{*}} \times A(\bar{Z}) \equiv\left(\frac{\partial}{\partial \bar{z}_{1}}, \frac{\partial}{\partial \bar{z}_{2}}, \cdots, \frac{\partial}{\partial \bar{z}_{n}}\right)^{\prime} \times A(\bar{Z}) .
$$

Throughout this paper, vectors and matrices marked with the symbol ' or * denote the transposed or transposed conjugate vectors and matrices, respectively.

Let us put

$$
T_{D}(Z, \bar{X}) \equiv \frac{\partial^{2}}{\partial Z \partial X^{*}} \log k_{D}(Z, \bar{X}),
$$

then $d s^{2}=d Z^{*} T_{D}(Z, \bar{Z}) d Z$ is a Kähler metric of $D$ and $T_{D}(Z, \bar{Z})$ is positive definite. The quantities $k_{D}(Z, \bar{X})$ and $T_{D}(Z, \bar{X})$ play important roles in this paper.

\section{Bergman's minimum problems.}

Definition 1. (i) $\left(X_{0}, X_{1} ; P\right)_{D}$-class denotes the class of holomorphic vector functions

$$
W(Z) \equiv\left(w_{1}(Z), w_{2}(Z), \cdots, w_{n}(Z)\right)^{\prime}
$$

in a domain $D$ in the $Z \equiv\left(z_{1}, z_{2}, \cdots, z_{n}\right)^{\prime}$-space which satisfy, at a nonbranch point $P=\left(p_{1}, p_{2}, \cdots, p_{n}\right)^{\prime} \in D$, the following initial conditions:

$$
W(P)=X_{0}, \quad \frac{d W(P)}{d Z}=X_{1}, \quad \operatorname{det} X_{1} \neq 0,
$$

where $X_{0}, X_{1}$ are $(n \times 1),(n \times n)$ constant matrices, respectively. And the class of image domains by the mappings belonging to the $\left(X_{0}, X_{1} ; P\right)_{D}$-class is called the $\left[X_{0}, X_{1} ; P\right]^{D}$-equivalent class.

(ii) $\left(x_{1} ; P\right)_{D}$-class denotes the class of holomorphic vector functions $W(Z)$ on $D$, which satisfy the following conditions:

$$
W(P)=0, \quad \operatorname{det} \frac{d W(P)}{d Z}=x_{1} \neq 0,
$$

where $x_{1}$ is a scalar constant. The equivalent class of image domains of $D$ obtained by the mappings $W(Z)$ belonging to the $\left(x_{1} ; P\right)_{D}$-class is called the $\left[x_{1} ; P\right]^{D}$-equivalent class. 
REMARK 1. Because $X_{0}$ denotes a parallel transformation and has no influence upon the situation of our theory, we will assume hereafter that $X_{0}=0$ without loss of generality. The $(1 ; P)_{D}$-class contains the $\left(0, I_{n} ; P\right)_{D}$-class, and therefore the $\left[0, I_{n} ; P\right]^{D}$-equivalent class is a subclass of the $[1 ; P]^{D}$-equivalent class.

THEOREM 1. (i) There exists the unique function belonging to the $\left(0, X_{1} ; P\right)_{D^{-}}$class which minimizes the integral

$$
\int_{D}|F(Z)|^{2} d v_{Z}=\int_{D} F^{*}(Z) F(Z) d v_{Z},
$$

where $F(Z) \in\left(0, X_{1} ; P\right)_{D}$-class and $d v_{Z}$ denotes the Euclidean volume element of the $Z$-space.

Let $M_{D}^{0 X_{1}}(Z, P)$ be the minimizing function for (13) and $\lambda_{D}^{0 X_{1}}(P)$ the minimum value of (13) for $M_{D}^{0 X_{1}}(Z, P)$, where $P$ is not on a branch manifold on $D$. Writing them as matrices, we have

$$
M_{D}^{o X_{1}}(Z, P)=\left(0, X_{1}\right)\left(H_{D}(P, \bar{P})\right)^{-1} L_{D}(Z, \bar{P}),
$$

and

$$
\lambda_{D}^{0 X_{1}}(P)=\operatorname{Spur}\left[\left(0, X_{1}\right)\left(H_{D}(P, \bar{P})\right)^{-1}\left(0, X_{1}\right)^{*}\right],
$$

where

$$
\begin{aligned}
& H_{D}(P, \bar{P})=\left(\begin{array}{ll}
k_{00^{*}} & k_{10^{*}} \\
k_{01^{*}} & k_{11^{*}}
\end{array}\right), \\
& L_{D}(Z, \bar{P})=\left(\begin{array}{l}
k_{D}(Z, \bar{P}) \\
k_{01^{*}}(Z, \bar{P})
\end{array}\right)
\end{aligned}
$$

and

$$
\begin{gathered}
k_{i j^{*}}(Z, \bar{X}) \equiv \frac{\partial^{i+j}}{\partial Z^{i} \partial X^{* j}} k_{D}(Z, \bar{X}), \\
k_{i j^{*}} \equiv k_{i j^{*}}(P, \bar{P}), \quad k_{00^{*}} \equiv k_{D}(P, \bar{P}) .
\end{gathered}
$$

(ii) For the $\left(x_{1} ; P\right)_{D}$-class, the minimizing function $W(Z)$ which minimizes the integral

$$
\int_{D}\left|\operatorname{det} \frac{d F(Z)}{d Z}\right|^{2} d v_{Z}, \quad F(Z) \in\left(x_{1} ; P\right)_{D^{-}} \text {class },
$$

satisfies

$$
\operatorname{det} \frac{d W(Z)}{d Z}=m_{D}^{x_{1}}(Z, P)=x_{1} k_{00 *}^{-1} k_{D}(Z, \bar{P})
$$


and the minimum value of (20) for (21) is

$$
\lambda_{D}^{x_{1}}(P)=x_{1} k_{00^{*}+\bar{x}_{1}}^{-1}=\frac{\left|x_{1}\right|^{2}}{k_{00^{*}}} .
$$

This theorem has been proved in [6] and our paper [10] for more general initial conditions. Therefore, we omit the proof of this theorem.

$m_{D}^{x_{1}}(Z, P)$ in (21) is the special case of (14) for the class of scalar functions $f(Z)$ with the initial condition $f(P)=x_{1}$.

The minimizing functions $M_{D}^{X_{0} X_{1}}(Z, P), m_{D}^{x_{1}}(Z, P)$ are relative invariant under any holomorphic mapping $W(Z)(\operatorname{det}(d W(P)) / d Z \neq 0)$ [3; p. 503], [4; pp. 765-770]; that is, putting $\Delta=W(D)$,

$$
\begin{gathered}
M_{D}^{X_{1} X_{0}}(Z, P)=M_{\Delta}^{Y_{1} Y_{0}}(W(Z), W(P)) \operatorname{det} \frac{d W(Z)}{d Z}, \\
m_{D}^{x_{1}}(Z, P)=m_{\Delta}^{y_{1}}(W(Z), W(P)) \operatorname{det} \frac{d W(Z)}{d Z},
\end{gathered}
$$

where $Y_{\nu}(\nu=0,1)$ and $y_{1}$ are determined for the function $G(W)$ such that $G(W(Z)) \operatorname{det}(d W(Z) / d Z)$ belongs to the $\left(X_{0}, X_{1} ; P\right)_{D}$-class by the system of equations

$$
\begin{gathered}
\left.\frac{d^{\nu}}{d Z^{\nu}}\left(G(W(Z)) \operatorname{det} \frac{d W(Z)}{d Z}\right)\right|_{Z=P}=X_{\nu}(\nu=0,1), \\
G^{(\nu)}(Q)=Y_{\nu}(\nu=0,1), \quad Q=W(P)
\end{gathered}
$$

and

$$
x_{1}=y_{1} \operatorname{det} \frac{d W(P)}{d Z}
$$

and $P$ is not on a branch manifold.

The system (25) has one and only one solution, because $\operatorname{det}(d W(P) / d Z) \neq 0$.

These minimum problems were treated originally by S. Bergman [1].

3. Minimal domains, representative domains and moment minimal domains.

DeFinition 2. Let $W=W(Z) \equiv\left(w_{1}(Z), w_{2}(Z), \cdots, w_{n}(Z)\right)^{\prime}$ be a holomorphic mapping of $D$ in $C^{n}$ belonging to the $(1 ; P)_{D}$-class and making (20) a minimum. The image domain $\Delta_{W}$ of $D$ under $W(Z)$ is called a (Bergman) minimal domain of $[1 ; P]^{D}$-equivalent class with center at $W(P)=0$ and $W(Z)$ a minimal function. 
The next theorem is well known [4], [10].

THEOREM 2. (i) If $W(Z)$ is a minimal function, then

$$
\operatorname{det} \frac{d W(Z)}{d Z}=m_{D}^{1}(Z, P) \equiv \frac{k_{D}(Z, \bar{P})}{k_{D}(P, \bar{P})} \in \mathscr{L}^{2}(D) .
$$

(ii) A domain $D$ is a minimal domain of the $[1 ; P]^{D}$-equivalent class with center at $P \in D$ if and only if

$$
m_{D}^{1}(Z, P)=1 \text { for } Z \in D,
$$

that is,

$$
k_{D}(Z, \bar{P})=k_{D}(P, \bar{P}) \quad \text { for } \quad Z \in D .
$$

(iii) $A$ minimal domain $D$ with center at $P$ is characterized by the following property: Any holomorphic mapping $F(Z)$ belonging to the $(1 ; P)_{D}$-class maps $D$ onto a domain whose volume is not less than the volume of $D$.

REMARK 2. A minimal function which minimizes the integral (20) is not uniquely determined. One of the minimal functions belonging to the $\left(0, I_{n} ; P\right)_{D}$-class $\left(\subset(1 ; P)_{D}\right.$-class $)$ is, for instance, given by

$$
W(Z) \equiv\left(\begin{array}{c}
w_{1}(Z) \\
w_{2}(Z) \\
\vdots \\
w_{n}(Z)
\end{array}\right)=\left(\begin{array}{c}
\int_{p_{1}}^{z_{1}} m_{D}^{1}\left(t, z_{2}, \cdots, z_{n} ; P\right) d t+f\left(z_{2}, \cdots, z_{n}\right) \\
z_{2}-p_{2} \\
\vdots \\
z_{n}-p_{n}
\end{array}\right),
$$

where $f\left(z_{2}, \cdots, z_{n}\right)$ is an arbitrary holomorphic scalar function of $\left(z_{2}, \cdots, z_{n}\right)$ and is equal to 0 at $Z=P=\left(p_{1}, p_{2}, \cdots, p_{n}\right)^{\prime}[5$, p. 230].

THEOREM 3. The function

$$
W_{D}(Z, P) \equiv \frac{M_{D}^{\text {oIn }}(Z, P)}{m_{D}^{1}(Z, P)} \in\left(0, I_{n} ; P\right)_{D^{-}} \text {class }
$$

is absolutely invariant under any mapping belonging to the $\left(0, I_{n} ; P\right)_{D^{-}}$ class.

Proof. This theorem is easily obtained by (23), (24). If $X_{0}=$ $(0, \cdots, 0)^{\prime}=0, X_{1}=I_{n}$, then we have $Y_{0}=0$ and $Y_{1}=I_{n}$ for any $F(Z) \in\left(0, I_{n} ; P\right)_{D}$-class by direct calculations (using (6), (7) and (8)).

Definition 3. The image domain $\Delta_{W}=W_{D}(D, P)$ is called the (Bergman) representative domain of the $\left[0, I_{n} ; P\right]^{D}$-equivalent class 
with center at the origin, and $W_{D}(Z, P)$ the representative function.

The next theorem is known [8], [9].

THEOREM 4. (i) A domain $D$ is a representative domain with center at $P$ if and only if

$$
\frac{M_{D}^{0 I}(Z, P)}{m_{D}^{1}(Z, P)}=Z-P, \quad Z \in D
$$

that is,

$$
T_{D}(Z, \bar{P})=T_{D}(P, \bar{P}), \quad Z \in D .
$$

(ii) $A$ domain $D$ is a minimal and also a representative domain with the same center at $P$ if and only if

$$
M_{D}^{0 I}(Z, P)=Z-P \quad \text { and } \quad m_{D}^{1}(Z, P)=1, \quad Z \in D,
$$

that is,

$$
k_{11^{*}}(Z, \bar{P})=k_{1^{*}}(P, \bar{P}) \quad \text { and } \quad k_{D}(Z, \bar{P})=k_{D}(P, \bar{P}), \quad Z \in D .
$$

In the above, $P$ denotes a parallel transformation of $D$. Therefore we may take $P=0$, without loss of generality. We shall take $P=0$ hereafter.

Definition 4. The minimizing function $W(Z) \in\left(0, I_{n} ; 0\right)_{D}$-class which minimizes the integral

$$
I=\int_{\Delta} F^{*}(Z) F(Z) d v_{F},
$$

where $F(Z) \in\left(0, I_{n} ; 0\right)_{D}$-class and $\Delta=F(D)$, is called the minimal function of moment of inertia (denoted as the moment minimal function of the $\left(0, I_{n} ; 0\right)_{D}$-class $)$, and the image domain $\Delta_{W}=W(D)$ the moment minimal domain of the $\left[0, I_{n} ; 0\right]^{D}$-equivalent class with center at the origin.

THEOREM 5. (i) The moment minimal function $W(Z)$ of the $\left(0, I_{n} ; 0\right)_{D^{-}}$class, if it exists satisfies

$$
W(Z) \operatorname{det} \frac{d W(Z)}{d Z}=M_{D}^{0 I} n(Z, 0) .
$$

The holomorphic function $W(Z)$ which satisfies (35), if it exists, is determined uniquely by the initial condition that $W(Z)$ belongs to the $\left(0, I_{n} ; 0\right)_{D}$-class.

(ii) $A$ domain $D$ is the moment minimal domain of the $\left[0, I_{n} ; 0\right]^{D}$-equivalent class if and only if 


$$
M_{D}^{o I}(Z, 0)=Z \text { for } Z \in D,
$$

that is, by the notation of (10)

$$
T_{D}(0,0)=\frac{k_{00 *} k_{11^{*}}(Z, 0)-k_{01 *} k_{10^{*}}(Z, 0)}{k_{00^{*}}^{2}}, \quad Z \in D,
$$

where $k_{00^{*}}=k_{D}(0,0)$.

Proof. (i ) For a function $F(Z) \in\left(0, I_{n} ; 0\right)_{D}$-class,

$$
I=\int_{\Delta} F^{*}(Z) F(Z) d v_{F}=\int_{D} F^{*}(Z) F(Z)\left|\operatorname{det} \frac{d F(Z)}{d Z}\right|^{2} d v_{Z},
$$

where $\Delta=F(D)$. It is clear that $F(Z) \operatorname{det}(d F(Z) / d Z)$ belongs to the $\left(0, I_{n} ; 0\right)_{D}$-class, thus by Theorem 1 the minimizing function $W(Z)$, if it exists, satisfies (35).

Conversely, if $W(Z)$ satisfies (35), then for an arbitrary function $F(Z) \in\left(0, I_{n} ; 0\right)_{D}$-class, we have

$$
\begin{aligned}
\int_{F(D)} F^{*}(Z) F(Z) d v_{F} & =\int_{D} F^{*}(Z) F(Z)\left|\operatorname{det} \frac{d F(Z)}{d Z}\right|^{2} d v_{Z} \\
& =\int_{D}\left|F(Z) \operatorname{det} \frac{d F(Z)}{d Z}\right|^{2} d v_{Z} \geqq \int_{D}\left|M_{D}^{o I} n(Z, 0)\right|^{2} d v_{Z} \\
& =\int_{D}\left|W(Z) \operatorname{det} \frac{d W(Z)}{d Z}\right|^{2} d v_{Z}=\int_{W(D)}|W(Z)|^{2} d v_{W} .
\end{aligned}
$$

This shows that $W(Z)$ is a moment minimal function belonging to the $\left(0, I_{n} ; 0\right)_{D}$-class.

Now we shall prove the uniqueness of the moment minimal function which satisfies (35). For simplicity, without loss of generality, we treat the case of $W(Z)=(u(Z), v(Z))^{\prime}, Z=(x, y)^{\prime}$.

Suppose that there exists the unique function $W=W(Z)$, which satisfies (35) and belongs to the $\left(0, I_{2} ; 0\right)_{D}$-class, on the representative domain $D$, and let the function $Z=Z\left(Z^{\circ}\right)$ be the representative function (uniquely determined by Theorem 3 ) which maps $D_{0}$ (an arbitrary domain belonging to the $\left[0, I_{2} ; 0\right]^{D}$-equivalent class) onto the representative domain $D$, then we have

$$
W(Z) \operatorname{det} \frac{d W(Z)}{d Z}=W\left(Z\left(Z^{0}\right)\right) \operatorname{det} \frac{d W\left(Z\left(Z^{0}\right)\right)}{d Z^{0}} \operatorname{det} \frac{d Z^{0}}{d Z}
$$

On the other hand, by (23) we have

$$
M_{D}^{0 I_{2}}(Z, 0)=M_{D^{0}}^{0 I_{2}}\left(Z^{0}, 0\right) \operatorname{det} \frac{d Z^{0}}{d Z} .
$$

Thus from (35) we obtain the unique function $W\left(Z\left(Z^{0}\right)\right) \equiv W^{0}\left(Z^{0}\right)$ 
$\left(\varepsilon\left(0, I_{2} ; 0\right)_{D^{0}}\right.$-class $)$ which satisfies

$$
W^{0}\left(Z^{0}\right) \operatorname{det} \frac{d W^{0}\left(Z^{0}\right)}{d Z^{0}}=M_{D^{0}}^{0 I_{2}}\left(Z^{0}, 0\right) .
$$

This shows that in (35) we may assume a domain $D$ to be the representative domain with center at origin, without loss of generality.

Now, on the representative domain $D$ we have

$$
M_{D}^{0 I_{2}}(Z, 0)=Z m_{D}^{1}(Z, 0)=\left(x m_{D}^{1}(Z, 0), y m_{D}^{1}(Z, 0)\right)^{\prime}
$$

by Theorem 4 . Thus, in place of (35), we have

$$
u(Z) \operatorname{det} \frac{d W(Z)}{d Z}=x m_{D}^{1}(Z, 0), \quad v(Z) \operatorname{det} \frac{d W(Z)}{d Z}=y m_{D}^{1}(Z, 0) .
$$

As $W(0)=0$ and $d W(0) / d Z=I_{2}$, in the neighborhood of the origin we see that

$$
u(Z)=x \widetilde{u}(Z), \widetilde{u}(0)=1 ; \quad v(Z)=y \widetilde{v}(Z), \widetilde{v}(0)=1 ;
$$

and from (38) and (39) we have

$$
\widetilde{u}(Z)=\widetilde{v}(Z) .
$$

From (38) and (40), we obtain

$$
\widetilde{u}(Z)^{2}\left(\widetilde{u}(Z)+x \frac{\partial}{\partial x} \widetilde{u}(Z)+y \frac{\partial}{\partial y} \widetilde{u}(Z)\right)=m_{D}^{1}(Z, 0) .
$$

We may assume that

$$
\begin{gathered}
m_{D}^{1}(Z, 0)=1+\sum_{\alpha+\beta \geqq 1} c_{\alpha \beta} x^{\alpha} y^{\beta}, \\
\widetilde{u}(Z)=1+\sum_{\alpha+\beta \geqq 1} a_{\alpha \beta} x^{\alpha} y^{\beta},
\end{gathered}
$$

where $c_{\alpha \beta}(\alpha+\beta \geqq 1)$ are given coefficients and $a_{\alpha \beta}(\alpha+\beta \geqq 1)$ have to be determined. Substituting (42) and (43) into (41), we have

$$
\left(1+\sum_{\alpha+\beta \geqq 1} a_{\alpha \beta} x^{\alpha} y^{\beta}\right)^{2}\left(1+\sum_{\alpha+\beta \geqq 1}(\alpha+\beta+1) a_{\alpha \beta} x^{\alpha} y^{\beta}\right)=1+\sum_{\alpha+\beta \geqq 1} c_{\alpha \beta} x^{\alpha} y^{\beta} .
$$

In comparing the coefficients of $x^{\alpha} y^{\beta}$ on both sides, we obtain

$$
\begin{aligned}
& a_{10}=\frac{c_{10}}{4}, \quad a_{01}=\frac{c_{01}}{4}, \quad a_{20}=\frac{c_{20}}{5}-a_{10}^{2}=\frac{c_{20}}{5}-\frac{1}{16} c_{10}^{2}, \\
& a_{11}=\frac{1}{5}\left(c_{11}-\frac{5}{8} c_{10} c_{01}\right), \quad a_{02}=\frac{c_{02}}{5}-\frac{1}{16} c_{01}^{2} \text {, etc. }
\end{aligned}
$$

Generally, as each $a_{\alpha \beta}$ is a polynomial in $c_{\alpha \beta}$ and $a_{i j}(i+j<\alpha+\beta$, 
$i \leqq \alpha, j \leqq \beta)$, we can thus determine $a_{\alpha \beta}(\alpha+\beta \geqq 1)$ uniquely by recurrence.

If $W(Z)$ exists in the neighborhood of the origin, by the method of analytic continuation we can obtain tne unique holomorphic solution of (35) on $D$ except in a denumerable number of analytic segments of manifolds of complex dimensions $\leqq n-1$.

(ii) Let $D$ be a moment minimal domain. Putting

$$
W(Z) \equiv Z \in\left(0, I_{n} ; 0\right)_{D} \text {-class },
$$

by Theorem 5 (i) we have

$$
Z=M_{D}^{0 T_{n}}(Z, 0) \text { for } Z \in D \text {. }
$$

Conversely, if $Z=M_{D}^{o I_{n}}(Z, 0)$ for $Z \in D$, for any $F(Z) \in\left(0, I_{n} ; 0\right)_{D^{-}}$ class we have

$$
\begin{aligned}
I & =\int_{D} Z^{*} Z d v_{Z}=\int_{D} M_{D}^{0 I} I^{*}(Z, 0) M_{D}^{0 I}(Z, 0) d v_{Z} \\
& \leqq \int_{D} F^{*}(Z) \overline{\operatorname{det} \frac{d F(Z)}{d Z}} F(Z) \operatorname{det} \frac{d F(Z)}{d Z} d v_{Z}=\int_{F(D)} F^{*}(Z) F(Z) d v_{F},
\end{aligned}
$$

because $F(Z) \operatorname{det}(d F(Z) / d Z)$ belongs to the $\left(0, I_{n} ; 0\right)_{D}$-class. This shows that $D$ is a moment minimal domain.

From (ii), if $D$ is a moment minimal domain, then

$$
\begin{aligned}
Z & =M_{D}^{0 I}(Z, 0) \equiv\left(0, I_{n}\right)\left(\begin{array}{ll}
k_{00^{*}} & k_{10^{*}} \\
k_{01^{*}} & k_{11^{*}}
\end{array}\right)^{-1}\left(\begin{array}{l}
k_{D}(Z, 0) \\
k_{01^{*}}(Z, 0)
\end{array}\right) \\
& \equiv T_{D}^{-1}(0,0) \frac{k_{00^{*}} k_{01^{*}}(Z, 0)-k_{01^{*}} k_{D}(Z, 0)}{k_{00^{*}}^{2}}
\end{aligned}
$$

where $T_{D}(0,0)=\left(k_{00^{*}} k_{11^{*}}-k_{01^{*}} k_{10^{*}}\right) / k_{00^{*}}^{2}$. Differentiating both sides of the above, we have (37). The converse is proved by integration.

REMARK 3. At the origin the condition (37) is satisfied by an arbitrary domain, but in general it may not hold on $D$.

ExAmple 1. In the case of a complex variable, (35) is reduced to

$$
w(z) \frac{d w(z)}{d z}=m_{D}^{01}(z, 0) .
$$

Integrating the above, we have

$$
\int_{0}^{w} w d w=\int_{0}^{z} m_{D}^{01}(z, 0) d z,
$$

consequently 


$$
\frac{w^{2}}{2}=\frac{1}{k_{00^{*}}^{2} T_{D}(0,0)} \int_{0}^{z}\left(k_{00^{*}} k_{01^{*}}(z, 0)-k_{01^{*}} k_{D}(z, 0)\right) d z
$$

Thus the moment minimal function $w=w(z)$ is a two-valued function, but from the initial condition of $w(z)$ (belonging to the $(0,1 ; 0)_{D^{-}}$ class) the only one moment minimal function is determined.

If $D$ is the unit circle, by [1]

$$
k_{D}(z, \bar{t})=\frac{1}{\pi(1-z \bar{t})^{2}} .
$$

By simple calculations we have

$$
\begin{aligned}
& k_{D}(z, 0)=\frac{1}{\pi}=k_{00^{*}}, \\
& k_{01^{*}}(z, 0)=-\frac{2}{\pi} z, \quad k_{01^{*}}(0,0)=0, \\
& T_{D}(0,0)=k_{11^{*}} / k_{00^{*}}=\pi\left(-\frac{2}{\pi}\right)=-2,
\end{aligned}
$$

and hence

$$
\int_{0}^{z} m_{D}^{01}(z, 0) d z=-\frac{\pi^{2}}{2} \int_{0}^{z} \frac{1}{\pi}\left(-\frac{2}{\pi} z\right) d z=\int_{0}^{z} z d z
$$

Therefore the unit circle is the moment minimal domain of the $[0,1 ; 0]^{D}$-equivalent class with center at the origin, and the moment minimal function is

$$
\frac{w^{2}}{2}=\frac{z^{2}}{2}, \text { i.e., } w=z \in(0,1 ; 0)_{D} \text {-class } .
$$

Thus for the unit circle the identity mapping is the only one moment minimal function belonging to the $(0,1 ; 0)_{D}$-class. Further the unit circle is an example of a minimal and also a representative domain with the same center at the origin from (28) and (31).

THEOREM 6. If $D$ is (a) a minimal and also (b) a representative domain with the same center at the origin, then $D$ is (c) a moment minimal domain with the same center.

In this theorem, we may exchange (a) or (b) for (c), respectively.

Proof. By Theorem 2, Theorem 4, and Theorem 5, necessary and sufficient conditions for (a), (b) and (c) are 


$$
m_{D}^{1}(Z, 0)=1, \frac{M_{D}^{0 I}(Z, 0)}{m_{D}^{1}(Z, 0)}=Z \text { and } M_{D}^{0 I} n(Z, 0)=Z \text { for } Z \in D,
$$

respectively. Therefore, any two of the above conditions are sufficient conditions for the remainder.

It is known that there exists a minimal but not representative domain with the same center, or a representative but not minimal domain with the same center [3], [4].

Example of minimal and also representative domains with the same center at the origin are Cartan irreducible symmetric domains or more generally bounded complete Carathéodory circular domains. Furthermore, they are simultaneously moment minimal domains with the same center [3], [10].

4. Extended class. Let us consider the $\left(0, X_{1} ; 0\right)_{D}$-class, where $X_{1}$ is an arbitrary constant $(n \times n)$ matrix satisfying $\operatorname{det} X_{1} \neq 0$.

Lemma 1. The $\left[0, X_{1} ; 0\right]^{D}$-equivalent class is equal to the $\left[0, I_{n} ; 0\right]^{4}-$ equivalent class, where $\Delta=F(D), F(Z) \in\left(0, X_{1} ; 0\right)_{D}$-class.

Proof. Let $W_{1}(Z), W_{2}(Z)$ belong to the $\left(0, X_{1} ; 0\right)_{D}$-class, and $\Delta_{W_{1}}, \Delta_{W_{2}}$ be the images of $D$ by the mappings $W_{1}(Z), W_{2}(Z)$, respectively. We may assume the existence of the mapping $\eta\left(W_{1}(Z)\right)=$ $W_{2}(Z)$ which maps $\Delta_{W_{1}}$ onto $\Delta_{W_{2}}$. Because from the hypothesis on domains, it may be assumed that $D$ is a bounded univalent domain and the existence of an inverse holomorphic mapping $Z=Z\left(W_{1}\right)$ in the neighborhood of $W_{1}=0$ follows from $\left.\operatorname{det}\left(d W_{1}(Z) / d Z\right)\right|_{z=0}=$ $\operatorname{det} X_{1} \neq 0$. Then we can define a function $Z=Z\left(W_{1}\right)$ on $\Delta_{W_{1}}$ by the method of analytic continuation. Put $\eta=\eta\left(W_{1}\right) \equiv W_{2}\left(Z\left(W_{1}\right)\right)$, then we have a desired holomorphic mapping $\eta\left(W_{1}\right)=W_{2}$ on $\Delta_{W_{1}}$, which maps $\Delta_{W_{1}}$ onto $\Delta_{W_{2}}$. Since $W_{1}(Z), W_{2}(Z)$ belong to the $\left(0, X_{1} ; 0\right)_{D^{-}}$ class, we have

$$
\eta\left(W_{1}(0)\right)=\eta(0)=W_{2}(0)=0 .
$$

Differentiating both sides of $\eta\left(W_{1}(Z)\right)=W_{2}(Z)$ with respect to $Z$ and putting $Z=0$ yields that

$$
\left.\left.\frac{d \eta\left(W_{1}\right)}{d W_{1}}\right|_{W_{1}=0} \frac{d W_{1}(Z)}{d Z}\right|_{Z=0}=\left.\frac{d W_{2}(Z)}{d Z}\right|_{Z=0},
$$

that is,

$$
\frac{d \eta(0)}{d W_{1}} X_{1}=X_{1}
$$


Thus we have

$$
\frac{d \eta(0)}{d W_{1}}=I_{n}
$$

From the above, we see that $\eta=\eta\left(W_{1}\right)$ belongs to the $\left(0, I_{n} ; 0\right)_{\Delta_{W_{1}}}{ }^{-}$ class. Thus we have the following:

THEOREM 7. The image domain $\triangle$ of $D$ under the mapping

$$
W=X_{1} \frac{M_{D}^{0 I} n(Z, 0)}{m_{D}^{1}(Z, 0)} \in\left(0, X_{1} ; 0\right)_{D} \text { class }
$$

is the representative domain of the $\left(0, I_{n} ; 0\right)_{4}$-equivalent class with center at the origin. Thus we call (44) the representative function of the $\left(0, X_{1} ; 0\right)_{D}$-class and $\Delta=W(D)$ the representative domain of the $\left[0, X_{1} ; 0\right]^{D}$-equivalent class.

Proof. It is clear from Lemma 1 that $W(Z)$ belongs to the $\left(0, X_{1} ; 0\right)_{D}$-class. By the holomorphic invariance of minimizing functions (see (23), (24)) we have

$$
W(Z)=X_{1} \frac{M_{D}^{o I}(Z, 0)}{m_{D}^{1}(Z, 0)}=X_{1} \frac{M_{\Delta}^{o Y_{1}}(W, 0) \operatorname{det} \frac{d W}{d Z}}{m_{\Delta}^{y_{1}}(W, 0) \operatorname{det} \frac{d W}{d Z}}=X_{1} \frac{M_{\Delta}^{o Y_{1}}(W, 0)}{m_{\Delta}^{y_{1}}(W, 0)} .
$$

Differentiating both sides of

$$
M_{D}^{0 I_{n}}(Z, 0)=M_{\Delta}^{0 Y_{1}}(W, 0) \operatorname{det} \frac{d W}{d Z}
$$

with respect to $Z$, and putting $Z=0$, we have

$$
\begin{aligned}
\frac{d}{d Z} M_{D}^{0 I_{n}}(0,0)=I_{n}= & \frac{d}{d W} M_{\Delta}^{\mathrm{OY}_{1}}(0,0) \frac{d W(0)}{d Z} \operatorname{det} \frac{d W(0)}{d Z} \\
& +M_{\Delta}^{0 Y_{1}}(0,0) \frac{d}{d Z} \operatorname{det} \frac{d W(0)}{d Z}=Y_{1} X_{1} \operatorname{det} X_{1},
\end{aligned}
$$

and thus

$$
Y_{1}=X_{1}^{-1} / \operatorname{det} X_{1}
$$

For

$$
m_{D}^{1}(Z, 0)=m_{\Delta}^{y_{1}}(W, 0) \operatorname{det} \frac{d W}{d Z}
$$

we have 


$$
y_{1}=1 / \operatorname{det} X_{1}
$$

as well as the above. Hence we obtain

$$
W=X_{1} \frac{M_{\Delta}^{0 Y_{1}}(W, 0)}{m_{\Delta}^{y_{1}}(W, 0)}=X_{1} \frac{Y_{1} M_{\Delta}^{0 I_{n}}(W, 0)}{y_{1} m_{\Delta}^{1}(W, 0)}=\frac{M_{\Delta}^{0 I_{n}}(W, 0)}{m_{\Delta}^{1}(W, 0)} .
$$

Recalling Theorem 4, this shows that the domain $\Delta=W(D)$ is the representative domain of the $\left[0, I_{n} ; 0\right]^{4}$-equivalent class.

CoROLlary 1. The unique mapping function $W=W(Z)$ which maps the representative domain $D$ of the $\left[0, I_{n} ; 0\right]^{D}$-equivalent class onto the representative domain $\triangle$ of the $\left[0, X_{1} ; 0\right]^{D}$-equivalent class (the $\left[0, I_{n} ; 0\right]^{4}$-equivalent class) with the same center at the origin, where $W(D)=\Delta$, is

$$
W=X_{1} Z
$$

Proof. If $D$ is the representative domain of $\left[0, I_{n} ; 0\right]^{D}$-equivalent class, then

$$
\frac{M_{D}^{0 I} n(Z, 0)}{m_{D}^{1}(Z, 0)}=Z \text { for } \quad Z \in D
$$

Thus by Theorem 7 we have the result.

THEOREM 8. If $F(Z)$ belongs to the $\left(x_{1} ; 0\right)_{D}$-class normalized by $F(0)=0$ and $\left|x_{1}\right|=1$, then the $\left[x_{1} ; 0\right]^{D}$-equivalent class becomes the $[1 ; 0]^{\Delta}$-equivalent class, where $F(D)=\Delta$. The image domain $\Delta_{W}=$ $W(D)$ by a mapping such that

$$
\operatorname{det} \frac{d W(Z)}{d Z}=x_{1} m_{D}^{1}(Z, 0) \text { for } Z \in D
$$

is a minimal domain of the $[1 ; 0]^{4}$-equivalent class with center at the origin. Hence we shall call $W(Z)$ a minimal function of the $\left[x_{1} ; 0\right]^{D}$-equivalent class with center at the origin.

Proof. For arbitrary two functions $\xi=F(Z)$ and $\eta=G(Z)$ belonging to the $\left(x_{1} ; 0\right)_{D}$-class normalized by $\left|x_{1}\right|=1$, we have the relation

$$
G(Z)=\eta(\xi)=\eta(F(Z))
$$

Differentiating both sides of the above with respect to $Z$, we have $d G(Z) / d Z=(d \eta / d \xi)(d F(Z) / d Z)$ and so 


$$
\operatorname{det} \frac{d G(Z)}{d Z}=\operatorname{det} \frac{d \eta}{d \xi} \operatorname{det} \frac{d F(Z)}{d Z} .
$$

Since $\operatorname{det}(d G(0) / d Z)=\operatorname{det}(d F(0) / d Z)=x_{1}$, we have

$$
\operatorname{det} \frac{d \eta(0)}{d \xi}=1 \text {. }
$$

Thus $\eta=\eta(\xi)$ belongs to the $[1 ; 0]^{\Delta}$-equivalent class, where $\Delta=F(D)$.

By (21), (24) and (46), for a minimizing function $W(Z) \in\left(x_{1} ; 0\right)_{D^{-}}$class we have

$$
\operatorname{det} \frac{d W(Z)}{d Z}=x_{1} m_{D}^{1}(Z, 0)=m_{\triangle_{W}}^{1}(W, 0) \operatorname{det} \frac{d W}{d Z},
$$

that is,

$$
m_{\Delta_{W}}^{1}(W, 0)=1 \text { for } W \in \Delta_{W} .
$$

This shows that $\Delta_{W}$ is a minimal domain of the $\left[x_{1} ; 0\right]^{D}$-equivalent class, i.e., the $[1 ; 0]^{4}$-equivalent class with center at the origin (by Theorem 2 and the above).

Existence of the mappings which satisfy the equation (46) is shown in Remark 2.

CoROllary 2. The mapping function $W(Z)$ (belonging to the $\left(x_{1} ; 0\right)_{D}$-class $)$ which maps a minimal domain $D$ of the $[1 ; 0]^{D}$ eqivalent class with center at the origin onto another minimal domain $\Delta$ of the $[1 ; 0]^{4}$-equivalent class, where $\Delta=W(D)$, satisfies the conditions

$$
W(0)=0, \quad \operatorname{det} \frac{d W(Z)}{d Z}=x_{1} \text { for } Z \in D,
$$

and vice versa. This mapping is a volume preserving one. One such mapping, for instance, is

$$
W=X_{1} Z, \quad \operatorname{det} X_{1}=x_{1} .
$$

Proof. By (21) and (24) we have

$$
m_{D}^{x_{1}}(Z, 0)=x_{1} m_{D}^{1}(Z, 0)=m_{\Delta}^{y_{1}}(W, 0) \operatorname{det} \frac{d W}{d Z}=y_{1} m_{\Delta}^{1}(W, 0) \operatorname{det} \frac{d W}{d Z},
$$

where $y_{1}=1$ since $W(Z)$ belongs to the $\left(x_{1} ; 0\right)_{D}$-class. Since $D$ and $\Delta$ are minimal domains of the $[1 ; 0]^{D}$-equivalent class and the $[1 ; 0]^{4}-$ equivalent class respectively, by (27) we have $m_{D}^{1}(Z, 0)=1$ for $Z \in D$ and $m_{\Delta}^{1}(W, 0)=1$ for $W \in \Delta$. Thus we obtain (47). The converse is 
clear from (24). The volume preserving property for $W(Z) \in\left(x_{1} ; 0\right)_{D^{-}}$ class, $\left|x_{1}\right|=1$, is the consequence of the following:

$$
\operatorname{vol}(\Delta)=\int_{\Delta} d v_{W}=\int_{D}\left|\operatorname{det} \frac{d W}{d Z}\right|^{2} d v_{Z}=\int_{D}\left|x_{1}\right|^{2} d v_{Z}=\int_{D} d v_{Z}=\operatorname{vol}(D) .
$$

It is clear that $W=X_{1} Z$, $\operatorname{det} X_{1}=x_{1}$, satisfies (46).

Hereafter we shall use the initial condition $(0, X ; 0)_{D}$ normalized by $|\operatorname{det} X|=1$. $\quad C_{X}$ denotes the set of $(n \times n)$ square constant matrices $X$ such that $|\operatorname{det} X|=1$. Let us introduce the wider class

$$
\mathscr{F}=\left\{\bigcup_{X}(0, X ; 0)_{D} \text {-class } \mid X \in C_{X}\right\} \text {. }
$$

This extended class contains the $\left(0, I_{n} ; 0\right)_{D}$-class and admits a group of transformations of $D$ with a subgroup of the $\left(0, I_{n} ; 0\right)_{D}$-class. $\mathscr{F}$ contains $\mathscr{F}_{1}=\left\{\mathrm{U}_{X_{1}}\left(0, X_{1} ; 0\right)_{D}\right.$-class $\left.\mid \operatorname{det} X_{1}=1\right\}$ as a subclass, too. It holds

$$
\mathscr{F} \supset \mathscr{F}_{1} \supset\left(0, I_{n} ; 0\right)_{D} \text {-class , }
$$

where $\mathscr{F}_{1}$ is equal to the $(1 ; 0)_{D}$-class (see Definition 2 ).

Theorem 9. (i) All minimal domains in the F्F-equivalent class have the same volume, which is equal to the volume of a minimal domain of the $\mathscr{F}_{1}$-equivalent class.

(ii) All representative domains in the F्F-equivalent class have the same volume, which is equal to the volume of the representative domain of the $\left[0, I_{n} ; 0\right]^{D}$-equivalent class.

Proof. (i ) By Theorem 8 and Corollary 2, the mapping function $W(Z)$ of a minimal domain $D$ of the $\mathscr{F}_{1}$-equivalent class onto an arbitrary minimal domain $\Delta$ in the $\mathscr{F}$-equivalent class satisfies

$$
\operatorname{det} \frac{d W(Z)}{d Z}=\operatorname{det} X m_{D}^{1}(Z, 0)=\operatorname{det} X, \quad X \in C_{X} .
$$

Therefore

$$
\begin{aligned}
\operatorname{vol}(\Delta) & =\int_{\Delta} d v_{W}=\int_{D}\left|\operatorname{det} \frac{d W(Z)}{d Z}\right|^{2} d v_{Z} \\
& =\int_{D}|\operatorname{det} X|^{2} d v_{Z}=\int_{D} d v_{Z}=\operatorname{vol}(D) .
\end{aligned}
$$

(ii) By Corollary 1, the mapping function $W(Z)$ of the representative domain $D$ of the $\left[0, I_{n} ; 0\right]^{D}$-equivalent class onto an arbitrary representative domain $\Delta$ in the $\mathscr{F}$-equivalent class is

$$
W=X Z, \quad X \in C_{X} \text {. }
$$


Thus we obtain

$$
\operatorname{vol}(\Delta)=\int_{\Delta} d v_{W}=\int_{D}\left|\operatorname{det} \frac{d W(Z)}{d Z}\right|^{2} d v_{Z}=\operatorname{vol}(D) .
$$

Corollary 3. Let $A, B$ and $C$ denote the sets of minimal domains, representative domains and moment minimal domains in the $\mathscr{F}$-equivalent class, respectively. If $A \cap B \neq \phi$, then $B=A \cap C$. (cf. Theorem 12).

Proof. An arbitrary domain $D \in A \cap B$ belongs to $C$ (by Theorem 6). By Theorem 9 (ii), $B \subset A$ holds. Thus we have $B \subset(A \cap C)$. On the other hand, it is clear that an arbitrary domain $D \in A \cap C$ belongs to $B$ (by Theorem 6). Thus we have the result.

THEOREM 10. Suppose in the $\mathscr{F}$-equivalent class no domain exists which is simultaneously a minimal and representative with respect to the same point (which can be chosen as the origin 0). Then there does not exist in that class a domain which is simultaneously a minimal and moment minimal. Further there does not exist a domain which is simultaneously a representative and moment minimal with respect to 0 .

Proof. Let $A, B$, and $C$ denote the sets mentioned in Corollary 3. By hypothesis we have $A \cap B=\phi$. If $A \cap C \neq \phi$ (or $B \cap C \neq \phi$ ), that is, if a domain $D$ belongs to $A \cap C$, then by Theorem 6, $D$ must belong to $B$. Thus $A \cap B \neq \phi$. This is a contradiction.

REMARK 4. If $A \cap C=\phi$, then $A \cap B=\phi$ and $B \cap C=\dot{\phi}$ hold, and if $B \cap C=\phi$, then $A \cap B=\phi$ and $A \cap C=\dot{\phi}$ hold.

THEOREM 11. (i) The moment minimal function $W(Z)$ of the $\left(0, X_{1} ; 0\right)_{D}$-class (where $X_{1} \in C_{X}$ is fixed) exists if and only if

$$
W(Z) \operatorname{det} \frac{d W(Z)}{d Z}=X_{1} \operatorname{det} X_{1} M_{D}^{0 I}(Z, 0) \quad \text { for } \quad Z \in D .
$$

(ii) Suppose that a moment minimal function $\eta(Z)$ of the extended class $\mathscr{F}$ exists, then

$$
\eta(Z) \operatorname{det} \frac{d \eta(Z)}{d Z}=\widetilde{X} \operatorname{det} \widetilde{X} M_{D}^{0 I} n(Z, 0),
$$

where

$$
\widetilde{X}=\left(\operatorname{det} \Omega_{D}\right)^{1 / 2 n} U \Omega_{D}^{-1 / 2} \equiv U T_{D}^{1 / 2}(0,0) /\left(\operatorname{det} T_{D}(0,0)\right)^{1 / 2 n}
$$


Here

$$
\Omega_{D}=\left(0, I_{n}\right)\left(H_{D}(0,0)\right)^{-1}\left(0, I_{n}\right)^{\prime}=T_{D}^{-1}(0,0) / k_{00^{*}}
$$

is a positive definite Hermitian matrix and $U$ is an arbitrary constant unitary matrix.

Let $D_{z}$ and $\Delta_{\eta}$ be the moment minimal domain of the $\left[0, I_{n} ; 0\right]^{D_{-}}$ equivalent class and a moment minimal domain of the $\mathscr{F}$-equivalent class respectively, then the following inequality holds:

$$
\int_{\Delta \eta} \eta^{*} \eta d v_{\eta} \leqq \int_{D_{Z}} Z^{*} Z d v_{Z}
$$

(iii) $\Delta$ is a moment minimal domain of the $\mathscr{F}$-equivalent class if and only if

$$
M_{\Delta}^{0 I_{n}}(\eta, 0)=\eta \quad \text { for } \quad \eta \in \Delta
$$

and

$$
\Omega_{\Delta}=\left(\operatorname{det} \Omega_{\Delta}\right)^{1 / n} I_{n}(\text { scalar matrix }),
$$

that is,

$$
T_{\Delta}(0,0)=\left(\operatorname{det} T_{\Delta}(0,0)\right)^{1 / n} I_{n}(\text { scalar matrix }) .
$$

Proof. (i ) From (23) and (45)

$$
M_{D}^{0 I_{n}}(Z, 0)=M_{d}^{0, X_{1}^{-1} / \operatorname{det} X_{1}}(W, 0) \operatorname{det} \frac{d W}{d Z}
$$

and

$$
\begin{aligned}
W(Z) \operatorname{det} \frac{d W(Z)}{d Z} & =X_{1} \operatorname{det} X_{1} M_{D}^{0 I} n(Z, 0) \\
& =X_{1} \operatorname{det} X_{1} M_{\Delta}^{0, X_{1}^{-1} / \operatorname{det} X_{1}}(W, 0) \operatorname{det} \frac{d W}{d Z}
\end{aligned}
$$

hold. Hence we have

$$
W(Z)=M_{\Delta}^{0 I_{n}}(W, 0) \text {. }
$$

This shows that, from (36), $\Delta=W(D)$ is the moment minimal domain of the $\left[0, I_{n} ; 0\right]^{A}$-equivalent class $\left(\left[0, X_{1} ; 0\right]^{D}\right.$-equivalent class).

Conversely, suppose that $W(Z)$ is the moment minimal function of the $\left(0, X_{1} ; 0\right)_{D}$-class. Since $W(Z) \operatorname{det}(d W / d Z)$ belongs to the $\left(0, X_{1} \operatorname{det} X_{1} ; 0\right)_{D}$-class, we have

$$
W(Z) \operatorname{det} \frac{d W}{d Z}=M_{D}^{0, X_{1} \operatorname{det} X_{1}}(Z, 0)=X_{1} \operatorname{det} X_{1} M_{D}^{0 I}(Z, 0) .
$$


(ii) This has been essentially proved in our paper [10]. (iii) By (2)

$$
\begin{array}{ll}
k_{D}(0,0)=k_{d}(0,0), & k_{D, 1^{*}}=k_{d, 10^{*}} \tilde{X}, \\
k_{D, 01^{*}}=\widetilde{X}^{*} k_{d, 01^{*}}, & k_{D, 11^{*}}=\widetilde{X}^{*} k_{d, 11^{*}} \tilde{X}
\end{array}
$$

hold, where $\Delta=\eta(D)$. Therefore we have

$$
\Omega_{\Delta}=\tilde{X} \Omega_{D} \widetilde{X}^{*}, \quad \widetilde{X}=\left(\operatorname{det} \Omega_{D}\right)^{1 / 2 n} U \Omega_{D}^{-1 / 2},
$$

and

$$
\begin{aligned}
\left(\operatorname{det} \Omega_{\Delta}\right)^{1 / n} \Omega_{\Delta}^{-1} & =\left[\operatorname{det}\left(\widetilde{X} \Omega_{D} \widetilde{X}^{*}\right)\right]^{1 / n}\left(\widetilde{X} \Omega_{D} \widetilde{X}^{*}\right)^{-1} \\
& =\left(\operatorname{det} \Omega_{D}\right)^{1 / n}\left(\operatorname{det} \Omega_{D}\right)^{-1 / n} I_{n}^{-1}=I_{n},
\end{aligned}
$$

because $\Omega_{D}^{*}=\Omega_{D}$.

If $\Delta=\eta(D)$ is a moment minimal domain of the $\mathscr{F}$-equivalent class, then $\Delta$ is the moment minimal domain of the $\left[0, I_{n} ; 0\right]^{\Delta}$-equivalent class. Furthermore from (36)

$$
M_{\Delta}^{0 I}(\eta, 0)=\eta \text { for } \eta \in \Delta,
$$

and the converse is true.

Corollary 4. (i) If $D_{Z}$ and $D_{W}$ are the moment minimal domains of the $\left[0, I_{n} ; 0\right]^{D_{-}-a n d}$ the $\left[0, I_{n} ; 0\right]^{D_{-}}$-equivalent classes, respectively, and $W=W(Z)$ (which maps $D_{Z}$ onto $D_{W}$ ) belongs to the $\left(0, X_{1} ; 0\right)_{D_{Z}}$-class, then the moment minimal function $W(Z)$ is uniquely given by

$$
W=W(Z)=X_{1} Z \text {. }
$$

(ii) If $\Delta_{\eta}$ is a moment minimal domain of the $\mathscr{F}$-equivalent class, then the moment minimal function $\eta=\eta(Z)$ (which maps $D_{Z}$ onto $\Delta_{\eta}$ ) is given by

$$
\eta=\eta(Z)=\widetilde{X} Z, \quad \widetilde{X}=\left(\operatorname{det} \Omega_{D_{Z}}\right)^{1 / 2 n} U \Omega_{D_{Z}}^{-1 / 2},
$$

where $\tilde{X}$ is defined in Theorem 11.

Proof. Since $D_{Z}$ is the moment minimal domain of the $\left[0, I_{n} ; 0\right]^{D_{Z_{-}}}$ equivalent class,

$$
M_{D_{Z}}^{\circ I_{n}}(Z, 0)=Z \text { for } Z \in D_{Z}
$$

holds. From Theorem 11 (ii) it follows that

$$
W(Z) \operatorname{det} \frac{d W(Z)}{d Z}=\left(\operatorname{det} X_{1}\right) X_{1} M_{D_{Z}}^{0_{n}}(Z, 0)=\left(\operatorname{det} X_{1}\right) X_{1} Z
$$


is a necessary and sufficient condition that $W(Z)$ (if it exists) is the moment minimal function of the $\left[0, X_{1} ; 0\right]^{D z}$-equivalent class. On the other hand, the function (58) satisfies (60) for $Z \in D_{Z}$. Thus from Theorem 5, (58) is the unique and holomorphic moment minimal function of the $\left[0, I_{n} ; 0\right]^{D Z}$-equivalent class.

(ii) As in (i), by (52) of Theorem 11, (59) is the unique moment minimal function of $\mathscr{F}$ up to the constant unitary matrices.

CoROllary 5. All moment minimal domains in the $\mathscr{F}$-equivalent class preserve their volumes (cf. Theorem 9 (i), (ii)).

Proof. By Corollary 4, if $W=W(Z)$ is the mapping which maps a moment minimal domain $D$ onto another moment minimal domain $\Delta$ in the $\mathscr{F}$-equivalent class, then we have

$$
W=W(Z)=X_{1} Z, \quad\left|\operatorname{det} X_{1}\right|=1 .
$$

Since

$$
\operatorname{vol}(\Delta)=\int_{\Delta} d v_{W}=\int_{D}\left|\operatorname{det} \frac{d W(Z)}{d Z}\right|^{2} d v_{Z}=\int_{D}\left|\operatorname{det} X_{1}\right|^{2} d v_{Z}=\operatorname{vol}(D),
$$

$W=W(Z)$ is a volume preserving mapping.

THEOREM 12. Let $A, B$ and $C$ denote the sets of minimal, representative and moment minimal domains in the $\mathscr{F}$-equivalent class, respectively. If any one of the relations $A \cap B \neq \phi, A \cap C \neq \phi$ and $B \cap C \neq \phi$ holds, then $A \supset B=C$ (cf. Corollary 3).

Proof. If $A \cap B \neq \phi$, then all domains belonging to $B$ are minimal domains by Theorem 9 (ii) and all domains belonging to $B$ belong to $C$ by Theorem 6. Further, by Corollary $5, C \subset A$ and hence by Theorem 6, $C \subset B$. Thus we obtain $A \supset B=C$. In the case $A \cap C \neq \phi$ or $B \cap C \neq \phi$, we have an analogous result.

EXAMPle 2. The Cartan domains (bounded irreducible symmetric domains of the four main types) are defined as follows: The first three types $D_{1}, D_{2}$ and $D_{3}$ are represented by

$$
\left\{Z \mid I_{n}-Z Z^{*}>0\right\}
$$

where $Z$ denotes an $(n \times m)$-matrix on $D_{1}, Z$ denotes an $(n \times n)$ symmetric matrix with diagonal elements multiplied by $\sqrt{2}$ on $D_{2}$ and $Z$ denotes an $(n \times n)$-skew-symmetric matrix on $D_{3}$. The fourth type $D_{4}$ is the set of $n$-dimensional row vectors $Z$ such that

$$
\left|Z Z^{\prime}\right|<1, \quad 1-2 Z Z^{*}+\left|Z Z^{\prime}\right|^{2}>0 \text {. }
$$


They are all minimal and also representative domains [7], [10]. Therefore by Theorem 6 they are moment minimal domains of the

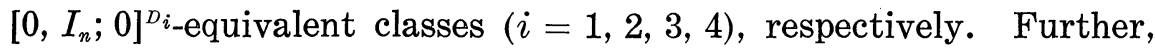

$$
T_{D_{i}}(0,0)=\left(k_{00^{*}} k_{11^{*}}-k_{01^{*}} k_{10^{*}}\right) / k_{00^{*}}^{2}=k_{11^{*}} / k_{00^{*}}=A_{i} \quad(i=1,2,3,4)
$$

hold, where

$$
A_{1}=(m+n) I_{m n}, \quad A_{2}=2(n+1) I_{n(n+1) / 2}, \quad A_{3}=2(n-1) I_{n(n-1) / 2}
$$

and $A_{4}=2 n I_{n}$ (property (57)). Therefore, they are moment minimal domains with respect to $\mathscr{F}\left(D_{i}\right)(i=1,2,3,4)$, respectively. Further in the $\mathscr{F}$-equivalent class of each one of Cartan domains, the set of all representative domains and the set of all moment minimal domains coincide and they are a subset of the set of minimal domains.

EXAMPLE 3. Bounded complete circular domains are minimal and also representative domains with center at the origin [3]. Thus each domain $D$ of them is the moment minimal domain with respect to the $\left[0, I_{n} ; 0\right]^{D}$-equivalent class, but it may not be a moment minimal domain of the $\mathscr{F}(D)$-equivalent class without property (57), which is equivalent to the "property $A$ " mentioned in J. Mitchell's paper [7] in the case of bounded complete circular domains.

\section{REFERENCES}

1. S. Bergman, The kernel function and conformal mapping, Amer. Math. Soc., New York, 1950.

2. L. K. Hua, Harmonic analysis of functions of several complex variables in the classical domains, Amer. Math. Soc. 6, 1963.

3. M. Maschler, Minimal domains and their Bergman kernel function, Pacific J. Math. 6 (1956).

4. - Classes of minimal and representative domains and their kernel functions, Pacific J. Math. 9 (1959).

5. H. Meschkowski, Hilbertsche Räume Mit Kernfunktion, Springer-Verlag, Berlin, 1962.

6. Y. Michiwaki, On the theory of analytic functions of several complex variables, Sci. Rep. Tokyo Kyoiku Daigaku Sec. A, 6 (1959).

7. J. Mitchell, Area and moment of inertia theorems for circular domains in $C^{n}$, Duke Math. J. 33 (1966).

8. T. Tsuboi, Bergman representative domains and minimal domains, Japanese J. Math. 29 (1959).

9. - n-representative domains and n-minimal domains in several complex variables, Dep. Math. Saitama Univ. Urawa (A) 4 (1961).

10. T. Tsuboi and S. Matsuura, Some canonical domains in $C^{n}$ and moment of inertia theorems, Duke Math. J. 36 (1969).

Received May 22, 1969.

Nagoya Institute of Technology

NAGOYA, JAPAN 


\section{PACIFIC JOURNAL OF MATHEMATICS}

\section{EDITORS}

H. SAMelson

Stanford University

Stanford, California 94305

\section{Richard PIERCE}

University of Washington

Seattle, Washington 98105
J. DugundjI

Department of Mathematics

University of Southern California

Los Angeles, California 90007

BASIL GoRDON*

University of California

Los Angeles, California 90024

\section{ASSOCIATE EDITORS}
E. F. BECKENBACH
B. H. NeumanN
F. WOLE
K. YosHIDA

\section{SUPPORTING INSTITUTIONS}

UNIVERSITY OF BRITISH COLUMBIA

CALIFORNIA INSTITUTE OF TECHNOLOGY

UNIVERSITY OF CALIFORNIA

MONTANA STATE UNIVERSITY

UNIVERSITY OF NEVADA

NEW MEXICO STATE UNIVERSITY

OREGON STATE UNIVERSITY

UNIVERSITY OF OREGON

OSAKA UNIVERSITY

UNIVERSITY OF SOUTHERN CALIFORNIA

\author{
STANFORD UNIVERSITY \\ UNIVERSITY OF TOKYO \\ UNIVERSITY OF UTAH \\ WASHINGTON STATE UNIVERSITY \\ UNIVERSITY OF WASHINGTON \\ * * * * \\ AMERICAN MATHEMATICAL SOCIETY \\ CHEVRON RESEARCH CORPORATION \\ TRW SYSTEMS \\ NAVAL WEAPONS CENTER
}

The Supporting Institutions listed above contribute to the cost of publication of this Journal, but they are not owners or publishers and have no responsibility for its content or policies.

Mathematical papers intended for publication in the Pacific Journal of Mathematics should be in typed form or offset-reproduced, (not dittoed), double spaced with large margins. Underline Greek letters in red, German in green, and script in blue. The first paragraph or two must be capable of being used separately as a synopsis of the entire paper. The editorial "we" must not be used in the synopsis, and items of the bibliography should not be cited there unless absolutely necessary, in which case they must be identified by author and Journal, rather than by item number. Manuscripts, in duplicate if possible, may be sent to any one of the four editors. Please classify according to the scheme of Math. Rev. 36, 1539-1546. All other communications to the editors should be addressed to the managing editor, Richard Arens, University of California, Los Angeles, California, 90024.

50 reprints are provided free for each article; additional copies may be obtained at cost in multiples of 50 .

The Pacific Journal of Mathematics is published monthly. Effective with Volume 16 the price per volume (3 numbers) is $\$ 8.00$; single issues, $\$ 3.00$. Special price for current issues to individual faculty members of supporting institutions and to individual members of the American Mathematical Society: $\$ 4.00$ per volume; single issues $\$ 1.50$. Back numbers are available.

Subscriptions, orders for back numbers, and changes of address should be sent to Pacific Journal of Mathematics, 103 Highland Boulevard, Berkeley, California, 94708.

PUBLISHED BY PACIFIC JOURNAL OF MATHEMATICS, A NON-PROFIT CORPORATION

Printed at Kokusai Bunken Insatsusha (International Academic Printing Co., Ltd.), 7-17, Fujimi 2-chome, Chiyoda-ku, Tokyo, Japan.

* Acting Managing Editor. 


\section{Pacific Journal of Mathematics}

\section{Vol. 33, No. $2 \quad$ April, 1970}

Raymond Balbes and Alfred Horn, Projective distributive lattices ....... 273

John Findley Berglund, On extending almost periodic functions ......... 281

Günter Krause, Admissible modules and a characterization of reduced left

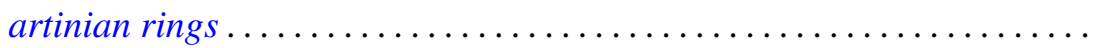

Edward Milton Landesman and Alan Cecil Lazer, Linear eigenvalues and a nonlinear boundary value problem ....................... 311

Anthony To-Ming Lau, Extremely amenable algebras ............... 329

Aldo Joram Lazar, Sections and subsets of simplexes .............. 337

Vincent Mancuso, Mesocompactness and related properties ............. 345

Edwin Leroy Marsden, Jr., The commutator and solvability in a generalized orthomodular lattice .................................. 357

Shozo Matsuura, Bergman kernel functions and the three types of canonical domains.......................................... 363

S. Mukhoti, Theorems on Cesàro summability of series .............. 385

Ngô Van Quê, Classes de Chern et théorème de Gauss-Bonnet ........... 393

Ralph Tyrrell Rockafellar, Generalized Hamiltonian equations for convex problems of Lagrange ................................ 411

Ken iti Sato, On dispersive operators in Banach lattices ............. 429

Charles Andrew Swanson, Comparison theorems for elliptic differential

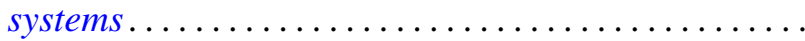

John Griggs Thompson, Nonsolvable finite groups all of whose local subgroups are solvable. II

David J. Winter, Cartan subalgebras of a Lie algebra and its ideals ... 